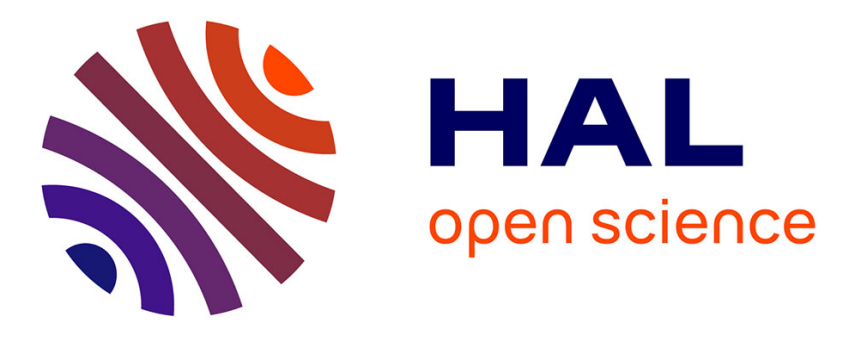

\title{
Regional Analysis of Left Ventricle Function using a Cardiac-Specific Polyaffine Motion Model
}

Kristin Mcleod, Christof Seiler, Nicolas Toussaint, Maxime Sermesant, Xavier Pennec

\section{- To cite this version:}

Kristin Mcleod, Christof Seiler, Nicolas Toussaint, Maxime Sermesant, Xavier Pennec. Regional Analysis of Left Ventricle Function using a Cardiac-Specific Polyaffine Motion Model. Functional Imaging and Modeling of the Heart 2013 (FIMH), Jun 2013, London, United Kingdom. pp.483-490, 10.1007/978-3-642-38899-6_57 . hal-00840042

\section{HAL Id: hal-00840042 https://hal.inria.fr/hal-00840042}

Submitted on 4 Jul 2013

HAL is a multi-disciplinary open access archive for the deposit and dissemination of scientific research documents, whether they are published or not. The documents may come from teaching and research institutions in France or abroad, or from public or private research centers.
L'archive ouverte pluridisciplinaire HAL, est destinée au dépôt et à la diffusion de documents scientifiques de niveau recherche, publiés ou non, émanant des établissements d'enseignement et de recherche français ou étrangers, des laboratoires publics ou privés. 


\title{
Regional Analysis of Left Ventricle Function using a Cardiac-Specific Polyaffine Motion Model
}

\author{
K. McLeod ${ }^{1}$, C. Seiler ${ }^{1,2}$, N. Toussaint ${ }^{3}$, M. Sermesant ${ }^{1}$, and X. Pennec ${ }^{1}$ \\ 1 INRIA Méditerranée, Asclepios Project, Sophia Antipolis, France \\ 2 Institute for Surgical Technology and Biomechanics, University of Bern, \\ Switzerland \\ 3 King's College London, Imaging Sciences, London, UK
}

\begin{abstract}
Given the complex dynamics of cardiac motion, understanding the motion for both normal and pathological cases can aid in understanding how different pathological conditions effect, and are affected by cardiac motion. Naturally, different regions of the left ventricle of the heart move in different ways depending on the location, with significantly different dynamics between the septal and free wall, and basal and apical regions. Therefore, studying the motion at a regional level can provide further information towards identifying abnormal regions for example. The 4D left ventricular motion of a given case was characterised by a low number of parameters at a region level using a cardiac specific polyaffine motion model. The motion was then studied at a regional level by analysing the computed affine transformation matrix of each region. This was used to examine the regional evolution of normal and pathological subjects over the cardiac cycle. The method was tested on 15 healthy volunteers with $4 \mathrm{D}$ ground truth landmarks and 5 pathological patients, all candidates for Cardiac Resynchronisation Therapy. Visually significant differences between normal and pathological subjects in terms of synchrony between the regions were obtained, which enables us to distinguish between healthy and unhealthy subjects. The results indicate that the method may be promising for analysing cardiac function.
\end{abstract}

\section{Introduction}

Cardiovascular disease still remains one of the worlds leading causes of mortality in the developed world, despite recent advances in the field. Research into congestive heart failure is increasingly providing new solutions to extend the life expectancy of heart failure patients. Given that cardiac asynchronous contraction is seen as a key characteristic of heart failure, Cardiac Resynchronisation Therapy (CRT) (where pacing leads are strategically implanted in a given region, or regions) can significantly reduce the risk of death in heart failure patients.

Common procedure for patients with heart disease is to scan the patient to obtain a 4D image sequence (using echocardiography, magnetic resonance (MR) 
or computed tomography (CT) scanners), for example, and / or electrocardiography (ECG) to measure heart rhythm. Image sequences allow to visualise in 3D the motion dynamics and to potentially identify thickened, ischemic, or scar regions, and asynchronous electrical conduction can be identified with ECG. Cardiac motion tracking from image sequences is a thoroughly studied area of research due to the useful insights it can provide for therapy planning (see [1],[2] and references therein for a sample of such methods).

The aim of the proposed method is two fold: first, differentiating between healthy and unhealthy subjects, and second, identifying abnormal regions of abnormal motion. To do so, a motion tracking scheme was required, with a small number of parameters describing the motion in order to obtain robust and reproducible measures. To this end, a method proposed by Mcleod et. al. [3] was applied, which has the advantage of incorporating anatomical and clinically meaningful prior information into the motion tracking through the use of anatomically oriented weight functions, and clinically relevant region definition. Using this method, the cardiac motion in 15 healthy volunteers was tracked along the cardiac cycle and quantitatively validated from ground truth annotated landmarks. As a clinical application of this study, the method was applied to 5 heart failure patients, to determine if there was a visible difference between the healthy and heart failure subjects. A preliminary analysis of the deformation parameters (the affine matrices) indicated that the method was able to detect meaningful differences between the two groups.

\section{Cardiac Motion Tracking with a Near-Incompressible Log-Domain Polyaffine Model}

Polyaffine LogDemons registration: Seiler et.al [4] proposed a method for estimating the polyaffine transformation between two images from a dense log demons deformation field by projecting the observed deformation field on a polyaffine space. The polyaffine model can be defined for points $x$ in (homogenous) Cartesian co-ordinates as $\log (T) \stackrel{\text { def }}{=} \log \left(\begin{array}{ll}A & t \\ 0 & 1\end{array}\right)=\left(\begin{array}{c}M \\ 0\end{array}\right)$ where $\log$ is a principal matrix logarithm, $A$ is the linear part of the transformation, $t$ its translation, and $M$ a $3 \times 4$ matrix. For each region the affine deformation fields parameterised by the $M_{i}$ matrices are fused to a global deformation field using the polyaffine model of Arsigny et. al [5]:

$$
\boldsymbol{v}_{\text {poly }}(x)=\sum_{i} \omega_{i}(x) M_{i} x
$$

where $\omega_{i}$ is a parameter controlling the weight of the $i^{t h}$ region for each voxel $x$. Eqn. 1 can be estimated by a linear least squares problem with the least squares error with respect to the observed velocity field $\boldsymbol{v}(x)$ (in this case computed using the LogDemons algorithm). 
Cardiac-specific features: Using this model, McLeod et. al [3] added an incompressibility penalisation and a neighbouring region regularisation to the model. Both terms were added in the least squares minimisation to obtain a linear penalised least squares error term given by:

$$
\bar{M}=\left(\Sigma \otimes I_{3}+\alpha V+\beta R\right)^{-1} \cdot \bar{B}
$$

In this way, parameters $\alpha$ and $\beta$ can be used to control the strength of the incompressibility penalisation and neighbouring region regularisation respectively. The final algorithm is summarised in Algorithm 1.

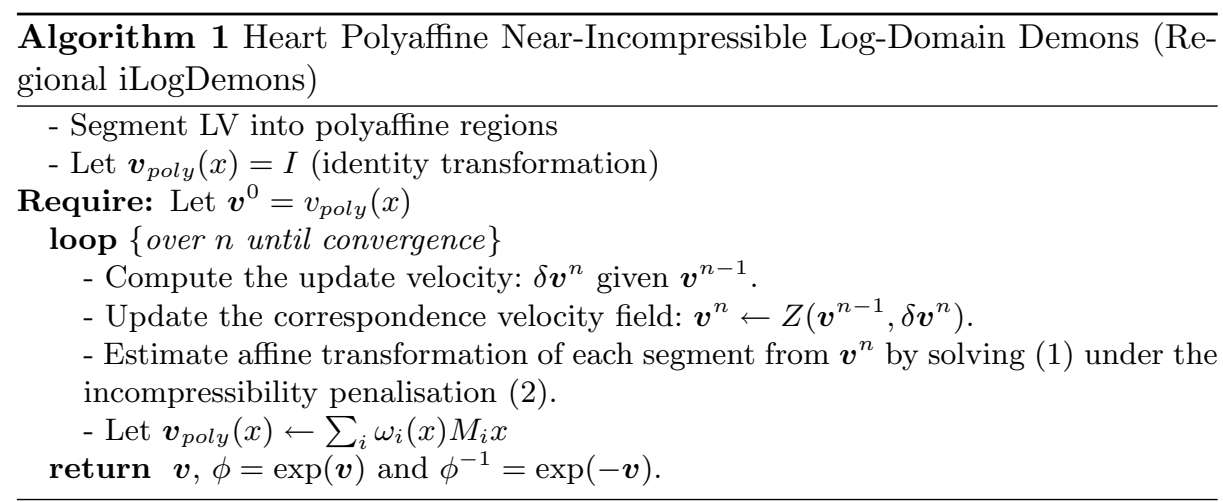

\section{Polyaffine Weight Function}

The weights $\omega_{i}(x)$ allow the fusion of the regional affine transformations to a global transformation. The fusion needs to be sufficiently smooth to ensure that the final fused transformation remains diffeomorphic. The weights can be defined by a simple Gaussian function as

$$
\omega_{i}(x)=-\exp \left(\frac{\kappa}{2}\left(x-\bar{x}_{i}\right)^{T} \phi_{i}^{(-1)}\left(x-\bar{x}_{i}\right)\right),
$$

with $\bar{x}_{i}$ the barycentre (centre point) of zone $i$ and $\phi_{i}$ the corresponding covariance matrix as in [4]. The parameter $\kappa$ is used to control the influence of neighbouring regions, with values less than one giving smoother transitions between the regions (see Fig. 1 left).

Using Eq. 3, each weight can be computed in standard Cartesian coordnates. These weights are sufficient in the case where the regions are arranged in straight lines, as in Fig. 1. However, for circular objects, more regions are needed to adequately capture the curvature of the object. 
Prolate spheroidal weights: Given the ellipsoidal shape of the left ventricle, the Prolate Spheroidal (PS) coordinate system has commonly been used to represent its function and architecture. The polyaffine weights in Eq 3 can be computed at each point in PS coordinates $(\mu, \nu, \phi)$ using the following equation (see Fig. 1 right):

$$
\begin{aligned}
& x=a \sinh \mu \sin \nu \cos \phi \\
& y=a \sinh \mu \sin \nu \sin \phi \\
& z=a \cosh \mu \cos \nu
\end{aligned}
$$

(see Fig. 1 right). Using the framework of Toussaint et. al [6], each point of the image can be converted to a point in PSS coordinates. The Gaussian function can then be evaluated at each point and written to an image on the original Cartesian grid.
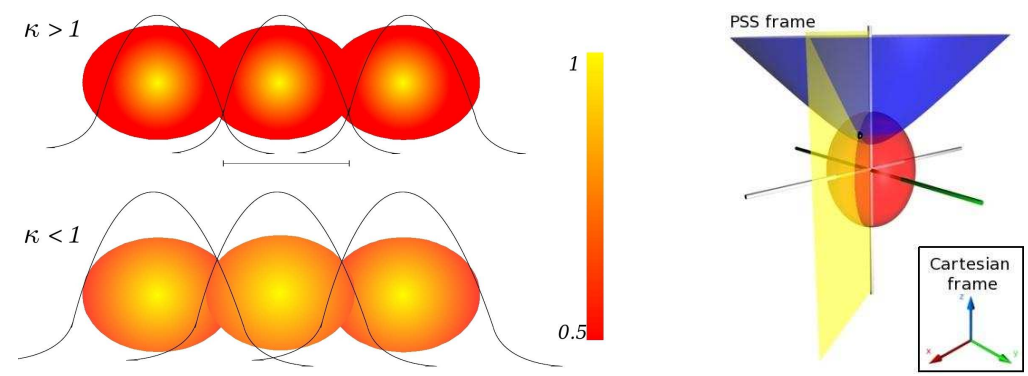

Fig. 1. Left - Illustration of the influence of $\kappa$ on the Gaussian function in Eq. 3 for three regions in a line. $\kappa>1$ gives more independent transformations, where $\kappa<1$ gives a smoother global motion. Right - The PSS grid $(\mu, \nu, \phi)$ with fixed $\mu$ in red (radial distance), fixed $\nu$ in blue (hyperbolic angle), and fixed $\phi$ in yellow (circumferential angle) (image adapted from www.wikipedia.org).

\section{Polyaffine Region Definition}

In order to apply the algorithm described in the previous section, a division of the left ventricle into sub-regions is required. Choice of these regions is dictated by the need for a sufficient number of regions $n$ to describe the motion ( $n$ close to 1 converges towards a global affine motion), while maintaining a low number of parameters describing the motion.

American Heart Association left ventricle segmentation: The standard LV segmentation given by the American Heart Association [7] provides an anatomically and clinically relevant delineation of 17 segments. It allows for a sufficient number of regions for the description of the LV motion and maintains a small number of parameters $(17 \times 12=204$ parameters per frame). 
A binary mask image of the left ventricle myocardium where the least squares minimisation is defined at the reference frame. This mask is then dilated to ensure that all trajectories along the cycle are contained within the mask. Each LV mask was then divided into 17 regions according to the AHA recommendations in a prolate spheroidal sense using a semi-automatic $\mathrm{C}++$ segmentation tool that required the input of three landmarks to define the base, apex, and LV-RV junction at the anterior wall.

\section{$5 \quad$ Left-Ventricular Motion Tracking}

\subsection{D Cardiac MR data sequences}

Healthy Volunteers with $4 D$ Landmarks: We illustrate these tools on 15 healthy adults ( 3 female, mean age $\pm \mathrm{SD}=28 \pm 5$ ) obtained from the STACOM 2011 MICCAI cardiac motion tracking challenge database [1]. Steady-state free precession magnetic resonance images were acquired using a 3T scanner (Philips Achieva System, Philips Healthcare) in the short axis view covering entirely both ventricles (12-16 slices; isotropic in-plane resolution:1.15 $\times 1.15 \mathrm{~mm}^{2}$ to $1.25 \times 1.25 \mathrm{~mm}^{2}$; slice thickness: $8 \mathrm{~mm}$; 30 frames). For each volunteer, $12 \mathrm{manu}-$ ally annotated landmarks were tracked along the cardiac cycle by two observers in 3D tagged MR image sequences of the same volunteers. These landmarks were transported to the cardiac MR sequences using the DICOM header information.

CRT Heart Failure Patients: To test the methods on pathological cases, we applied the described method to five adults with asynchronous contraction of the left ventricle. These patients were candidates for Cardiac Resynchorisation Therapy (CRT), all with a large QRS duration due to a Left Bundle Branch Block (LBBB). Steady-state free precession magnetic resonance images were acquired for each patient in the short axis view covering entirely both ventricles (12-16 slices; isotropic in-plane resolution: $1.42 \times 1.42 \mathrm{~mm}^{2}$ to $1.56 \times 1.56 \mathrm{~mm}^{2}$; slice thickness: 7-9mm; 30-40 frames).

\subsection{Quantitative Validation}

In order to quantitatively validate the tracking accuracy of the method, the same procedure was followed as described in [1]. The polyaffine model was applied to each of the 15 healthy volunteers. The incompressibility and penalisation parameters used were those given in [3], namely $\alpha=10, \beta=1$. The method was also applied with no incompressibility penalisation or regularisation $(\alpha=\beta=0)$. The tracking errors were computed at two time frames (end diastole (ED) and end systole (ES)), for both observers. The computed mean error for the PSS weights was 4.73 for no incompressibility or penalisation and 4.43 for the penalised and regularised case. This can be directly compared to the STACOM challenge methods with 3.14 for the TDFFD method and 4.78 for the iLogDemons method [1]. The errors are plotted in Fig. 2 (left). The penalisation and regularisation smooth 
the result to be more physiological reasonable without degrading the accuracy of the registration, as shown in Fig. 2 (center and right). These errors are consistent with those reported in [1].
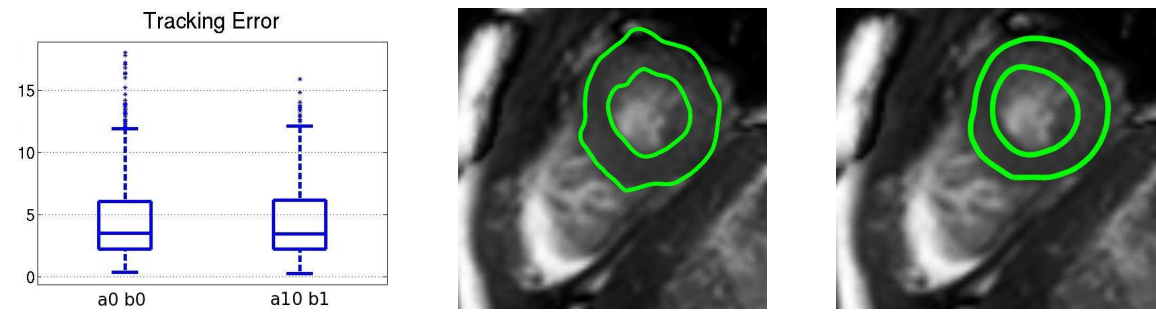

Fig. 2. Box plot of the tracking error with PSS weights (left) with no penalisation or regularisation (left column) and the penalised and regularised case $-\alpha=10, \beta=1$

(right column). Contour of the registration (in green) shown on one slice with no penalisation or regularisation (center), and with the penalised and regularised case (right). Adding the penalisation and regularisation to the computation gives a smoother registration.

\section{Regional Analysis of Polyaffine Parameters}

Since the transformation $T$ from one image to another is given in terms of the exponential of the affine matrix $M$ (see [8] for further details), i.e $T=$ $\exp (M)$, we can use the property derived from Lie Algebra for the Jacobian determinant: $\operatorname{det}(\exp (M))=\exp (\operatorname{tr}(M))$. Thus, we can analyse the trace of each matrix directly as a measure of volume change per region. Note that the incompressibility term is a penalisation rather than a hard constraint, since we don't want to force full incompressibility. Furthermore, in order to obtain full incompressibility, a larger number of regions would be needed. Therefore, we are interested in reducing, rather than forcing to zero, the compressibility. Plotting the trace per region along time for all the healthy volunteers shows a similar deformation at similar times for all the regions (see Fig. 3).

Plotting the same curves for the CRT patients shows abnormal behaviour between the regions compared with the normal cases (see Fig. 4). Patients 1, 3 and 4 have asynchrony between the regions, which can be expected given that these patients have ischemic regions. Patients 2 and 5 have Dilated Cardiomyopathy (DCM) resulting in reduced pumping function in the left ventricle.

Using Patient 4 as a specific case study (adult female with heart failure), the trace evolution can be analysed at the scar regions. For this patient, the scar location was segmented from the MR images. Significant mechanical asynchrony for the patient was confirmed from echocardiography, with left bundle branch block (LBBB). Two scars were observed, the first in regions 4 and 5 , the second in regions 9,10,14 and 15 (see Fig. 5 left). Plotting the trace separately for each scar 

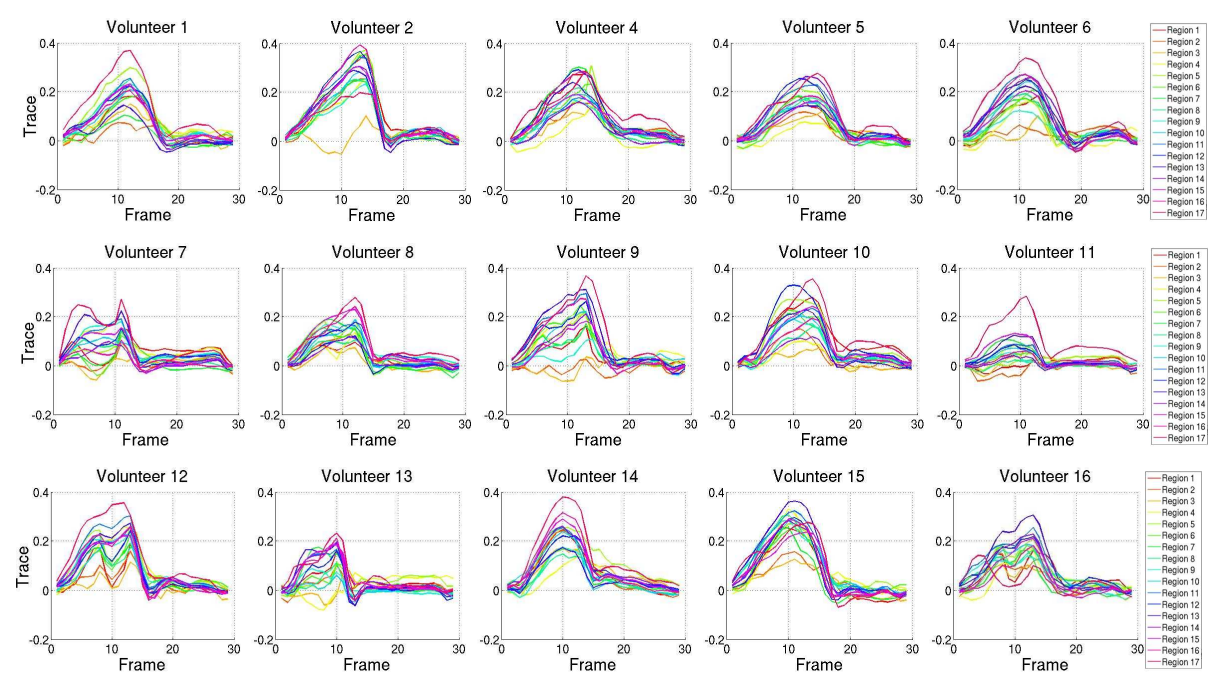

Fig. 3. Trace per region of each affine matrix for each of the healthy volunteers plotted over time. The figures show abnormal behaviour over time of the trace between all regions for all volunteers in comparison to the normal cases.

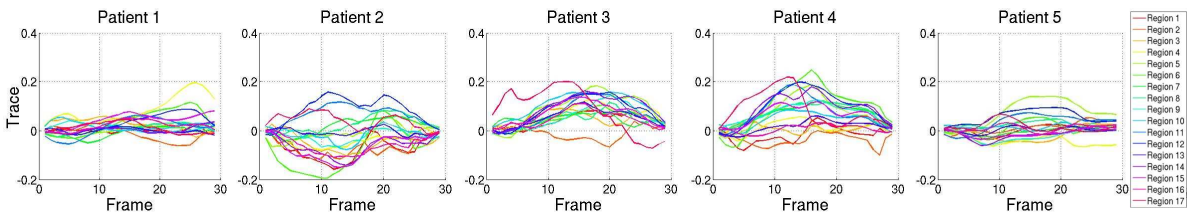

Fig. 4. Trace per region of each affine matrix for the CRT patients. Clearly, there is a asynchrony between the regions for all of the patients.

(see Fig. 5 centre and right), we see that there is a large difference in amplitude between regions 4 and 5 for the first scar, but with a single peak in the middle of the cycle, and smaller differences between the regions for the second scar (see Fig. 4). Since the second scar is divided amongst four regions, each region has only a small amount of scar, with mostly healthy tissue.

\section{Discussion and Future Work}

A recently proposed method for cardiac motion tracking was applied to a small database of 15 healthy and 5 heart failure subjects. This method presents the advantage of requiring a smaller number of parameters compared to previously reported methods. The validation on healthy subjects produced motion tracking results that agree well with literature, with a similar degree of error. As the deformation is described with few parameters, they can be used as bio-markers to differentiate between healthy and unhealthy subjects. The results suggest that the proposed method can be used for the comparison of cardiac motion patterns between groups and the identification of abnormal motion areas directly from 

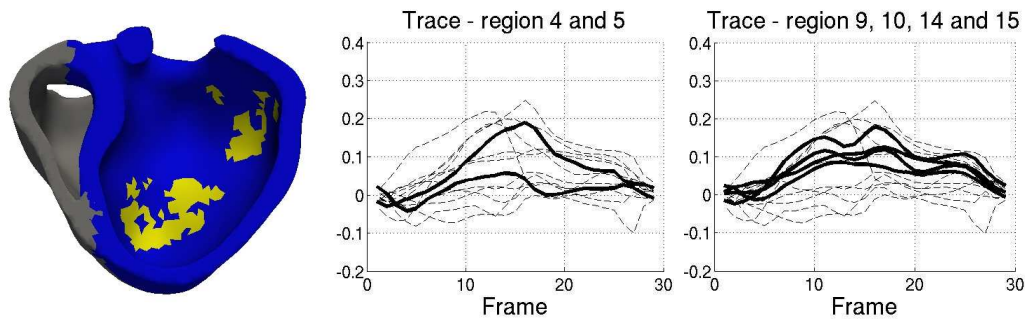

Fig. 5. Left: Bi-ventricle mesh of a LBBB patient sliced through to show the right ventricle (grey), left ventricle (blue) and scar location (yellow). Trace of the regions where one of the scars are located (centre), and trace of the regions where the other scar is located (right).

the images, without requiring any other additional measurements. It was shown that using this methods the motion parameters can be analysed at a regional level to study differences between regions of healthy or unhealthy tissue, though including the percentage of scar per region would provide more depth to this analysis to identify how much the scar region impacts the motion.

Acknowledgements: This project was partially funded by the Care4Me ITEA2 project and the Swiss National Science Foundation.

\section{References}

1. Tobon-Gomez, C., Craene, M.D., McLeod, K., Lautz, T., Shi, W., Hennemuth, A., Prakosa, A., Wang, H., Carr-White, G., Kapetanakis, S., Lutz, A., Rasche, V., Schaeffter, T., Butakoff, C., Friman, O., Mansi, T., Sermesant, M., Zhuang, X., Ourselin, S., Peitgen, H.O., Pennec, X., Razavi, R., Reuckert, D., Frangi, A., Rhode, K.: Benchmarking framework for myocardial tracking and deformation algorithms: An open access database. Med. Im. Anal. (2013)

2. Wang, H., Amini, A.: Cardiac motion and deformation recovery from MRI: a review. IEEE Trans. Med. Imag. 31(2) (2012)

3. McLeod, K., Seiler, C., Sermesant, M., Pennec, X.: A near-incompressible polyaffine motion model for cardiac function analysis. In: Proc. STACOM MICCAI workshop. LNCS, Springer (2012)

4. Seiler, C., Pennec, X., Reyes, M.: Capturing the multiscale anatomical shape variability with polyaffine transformation trees. Med. Im. Anal. (2012)

5. Arsigny, V., Commowick, O., Ayache, N., Pennec, X.: A fast and log-euclidean polyaffine framework for locally linear registration. J. Math. Im. and Vision 33(2) (2009)

6. Toussaint, N., Stoeck, C.T., Kozerke, S., Sermesant, M., Batchelor, P.G.: In-vivo human 3d cardiac fibre architecture: Reconstruction using curvilinear interpolation of diffusion tensor images. In: Proc. MICCAI'10. LNCS, Springer (2010)

7. Cerqueira, M., Weissman, N., Dilsizian, V., Jacobs, A., Kaul, S., Laskey, W., Pennell, D., Rumberger, J., Ryan, T., Verani, M.: Standardized myocardial segmentation and nomenclature for tomographic imaging of the heart. Circulation 105 (2002)

8. Commowick, O., Arsigny, V., Isambert, A., Costa, J., Dhermain, F., Bidault, F., Bondiau, P.Y., Ayache, N., Malandain, G.: An efficient locally affine framework for the smooth registration of anatomical structures. Med. Im. Anal. 12(4) (2008) 